\title{
ОПРЕДЕЛЕНИЕ ДЛИНЫ ПЕРЕХОДНО- СКОРОСТНОЙ ПОЛОСЫ НА ДОРОЖНЫХ РАЗВЯЗКАХ В РАЗНЫХ УРОВНЯХ
}

В работе представлен метод расчета длины переходно-скоростной полосы на дорожных развязках в разных уровнях при разгоне автомобилей. Увеличение интенсивности движения на автомобильных дорогах вызывает необходимость реконструкции автомобильных дорог, частью которой является строительство дорожных развязок в разных уровнях. Одним из основным элементов решения является длина переходно-скоростных полос при торможении и разгоне автомобилей. Переходно-скоростная полоса при торможении выполняет роль освобождения полосы движения на главной дороге и уменьшения скорости движения на переходно-скоростной полосе.

Переходно-скоростная полоса движения при разгоне автомобилей применяется для достижения средней скорости движения, равной скорости на основной дороге и обеспечивает вливания автомобилей с переходно-скоростной полосы (ПСП) в полосу движения на основной дороге. Основным показателем является изменение полосы движения с ПСП на полосу движения главной дороги, которое зависит от интенсивности движения на главной дороге и временного интервала между автомобилями. Выполнение манёвра автомобиля при смене полосы движения состоит из времени для его выполнения и длины участка дороги, на котором он выполняется. В работе представлены результаты исследования как времени на выполнение манёвра , так и длины участка дороги на котором он выполняется. Полученные результаты исследований применяются как для проектирования дорожных развязок в разных уровнях, так и для обоснования её типа. При значительной интенсивности движения на главной дороге возникает необходимость удлинения ПСП, а при невозможности въезда строительство вспомогательной полосы движения.

Ключевые слова: дорожные развязки в разных уровнях, переходно-скоростная полоса, полоса движения, временной интервал движения.

\section{1. ВВЕДЕНИЕ}

Чтобы обеспечить нормальные условия съезда и вхождения второстепенного потока в основной, проектируют переходно-скоростную полосу разгона, которая позволяет получить такое смещение «фаз» в пространственном распределении интервалов между автомобилями основного и второстепенного потоков, который создаёт условия для безопасного вхождения второго в первый. Поэтому для дальнейших расчетов за основу берём длину переходно-скоростной полосы. Схема переходно-скоростных полосы показано на рисунке 1.

\footnotetext{
1 Довгополюк Людмила - магистр, ассистент кафедры проектирования дорог, геодезии и землеустройства Киевского Национального транспортного университета ул. Суворова 1, Киев 10110, Украина, katze_@ukr.net
} 


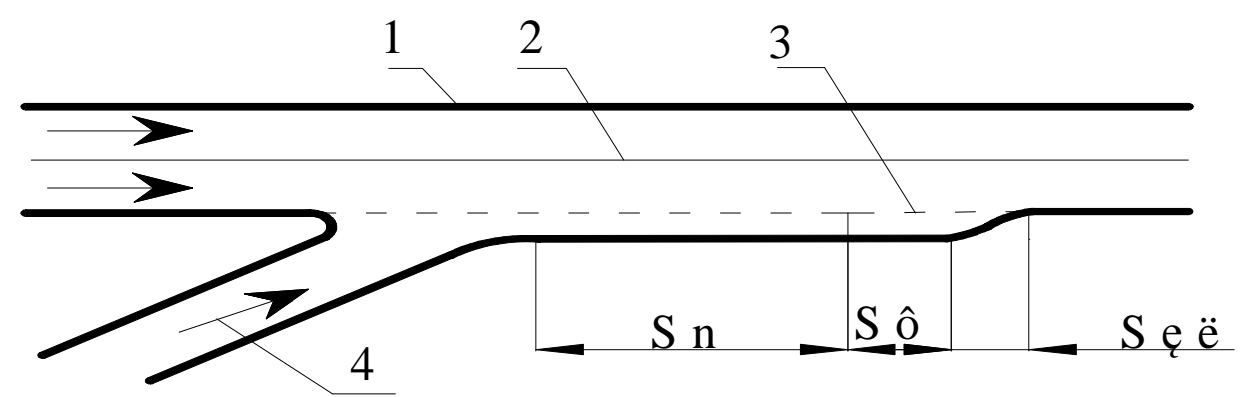

Рис. 1. Переходно-скоростная полоса для выхода со съезда (1 - краевая разметка, 2 сплошная разметка 3 - прерывистая разметка, 4 - съезд).

Переходно-скоростная полоса разгона состоит из трех участков: клина разгона, фазового участка и шлюза ускорения:

$$
L_{B И X}=S_{n}+S_{\Phi}+S_{K J}
$$

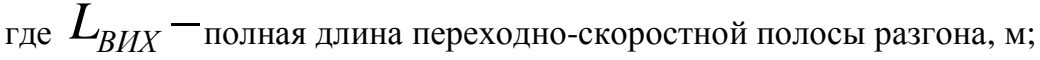

$S_{K л}$ - длина клина отгона, м;

$S_{n}$ - длина шлюза ускорения, м;

$S_{\Phi}-$ длина фазового участка, м.

Минимальная длина скоростного шлюза:

$$
S_{n}=\frac{v_{\Gamma}^{2}-v_{c}^{2}}{2 \cdot\left(a_{c}\right)}
$$

где - $v_{\Gamma}$ - скорость движения на крайней полосе главной дороги, м / с;

$v_{c}$ - скорость движения автомобилей на съезд, м / си;

$a_{c}$-среднее ускорение при разгоне автомобиля, м / с.

Минимальная длина фазовой участка определяется по формуле:

$$
S_{\phi}=v_{0} \cdot \frac{n}{\mathrm{~A}_{0}}
$$

где $v_{0}-$ средняя скорость движения, на которой выезжают автомобили со съезда;

$n$-среднее количество автомобилей в очереди;

$\mathrm{A}_{0}$ - пропускная способность крайней полосы движения на основной дороге, на которую выезжает со съезда автомобиль. 


$$
\begin{aligned}
& n=\psi \cdot \frac{1+n_{0} \frac{\psi_{0}}{\psi}}{1-\psi_{0}-\psi} \\
& \psi=\frac{N_{\mathrm{c}}}{\mathrm{A}_{0}-N_{0}} \\
& \psi_{0}=\frac{N_{0}}{\mathrm{~A}_{0}} \\
& n_{0}=\frac{\psi_{0}}{1-\psi_{0}}
\end{aligned}
$$

где $N_{\text {с }}$ - часовая интенсивность движения автомобилей на съезде, авто / ч;

$N_{0}$ - интенсивность движения на крайней полосе основной дороги, на которую выходят автомобили со съезда, авто / ч.

Необходимая длина клина отгона рассчитывается по формуле:

$$
S_{K J}=2 \times \sqrt{R_{0} \times b}
$$

где $R_{0}$ - минимальный радиус горизонтальной кривой без устройства виража на основной дороге, м;

$b$ - ширина переходно-скоростной полосы, м.

Даный метод не учитывает интервалы движения автомобилей на главной дороге, поэтому возникла необходимость разработки более точного метода.

\section{2. ИССЛЕДОВАНИЕ МАНЁВРА ВЛИВАНИЯ АВТОМОБИЛЕЙ НА ГЛАВНУЮ ДОРОГУ}

В зависимости от состава транспортного потока определяется интенсивность движения на главной дороге по крайней полосе движения и временной интервал движения.

Если интенсивность на второстепенной дороге будет больше, чем свободных интервалов на главной дороге $\left(\mathrm{N}_{\text {осн }}\right.$ на главной дороге), транспортный поток на второстепенной дороге не успеет выполнить манёвр вливания (то есть будут возникать задержки на переходно-скоростной полосе). В таком случае необходимо будет увеличивать длину переходно-скоростной полосы или устраивать дополнительную полосу движения.

Исследование было выполнено на дорожной развязке на объездной дороге г. Бровары Киевской области. Определялись скорость движения и длина участка дороги, необходимая для смены полосы движения.

Результаты экспериментальных данных для вливания в основной поток показаны на графиках. 


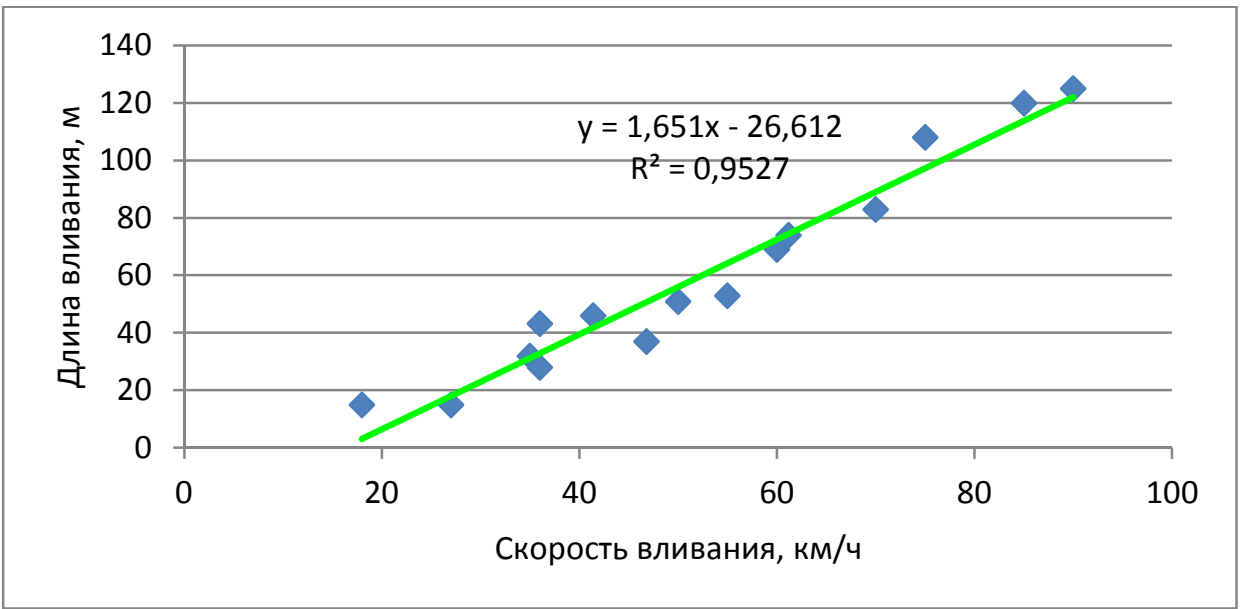

Рис. 2. Экспериментальные данные для вливания легковых автомобилей в транспортный поток $\left(l_{\text {авто }}=4\right.$ м)

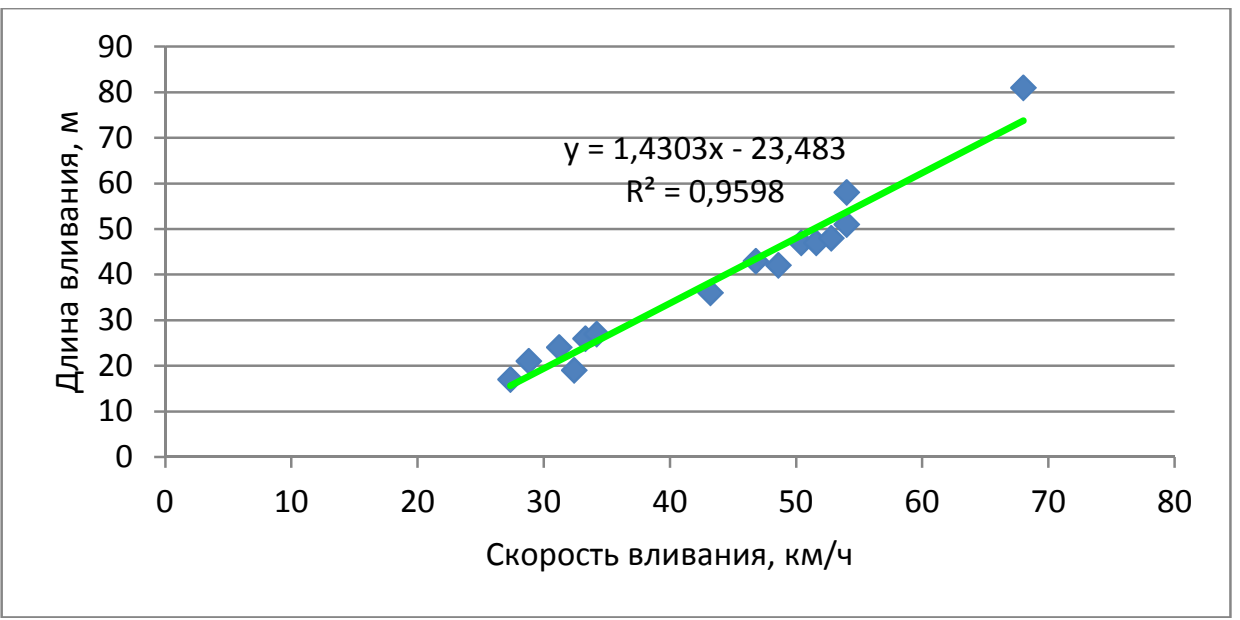

Рис. 3. Экспериментальные данные для вливания грузовых автомобилей в транспортный поток $\left(l_{\text {авто }}=7 \mathrm{M}\right)$ 


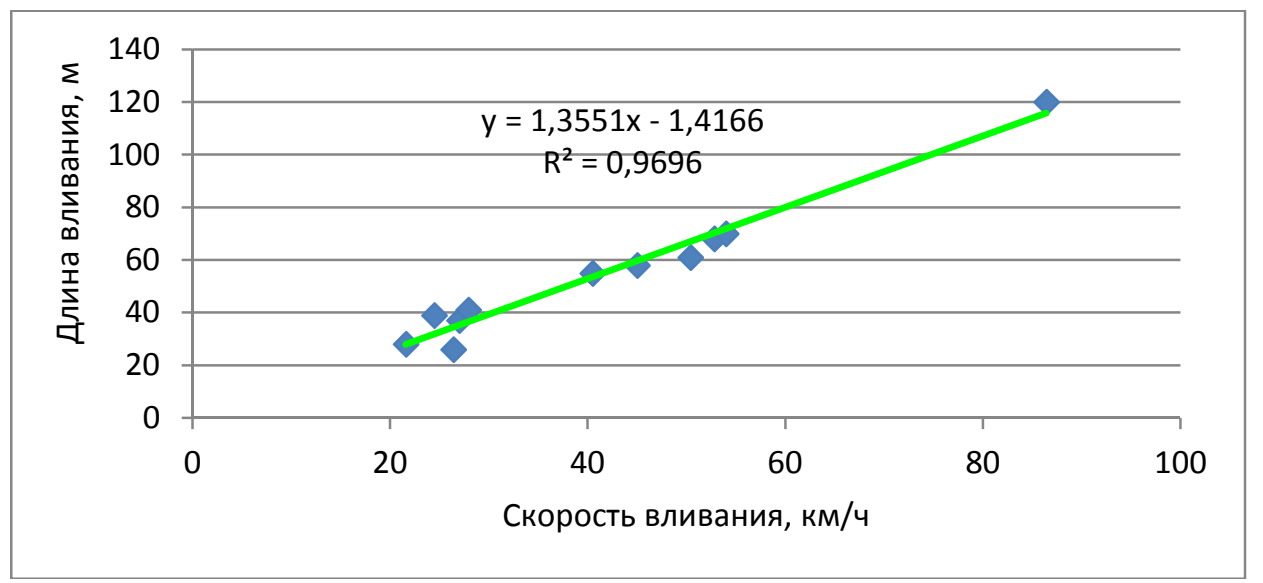

Рис. 4. Экспериментальные данные для вливания автопоездов в транспортный поток $\left(l_{\text {авто }}=12 \mathrm{M}\right)$

В конечном итоге, получена формула для определения времени, необходимого для выполнения манёвра в зависимости от скорости $(v)$ и состава транспортного потока $\left(l_{c p}\right.$ - средняя длина автомобиля):

$$
\begin{aligned}
& t=\left(0,0164 \times l_{c p}+0,0069\right) \times v^{2}-\left(0,0112 \times l_{c p}+0,4364\right) \times v+ \\
& +0,0276 \times l_{c p}+2,1475
\end{aligned}
$$

где $v$ - средняя скорость транспортного потока, (м/с).

\section{3. ИССЛЕДОВАНИЕ ВРЕМЕНИ СМЕНЫ ПОЛОСЫ ДВИЖЕНИЯ}

На основании выполненых исследований получено функциональные зависимости «интервал-интенсивность» на главной автомобильной дороге для определения времени, необходимого для выполнения манёвра вливания.

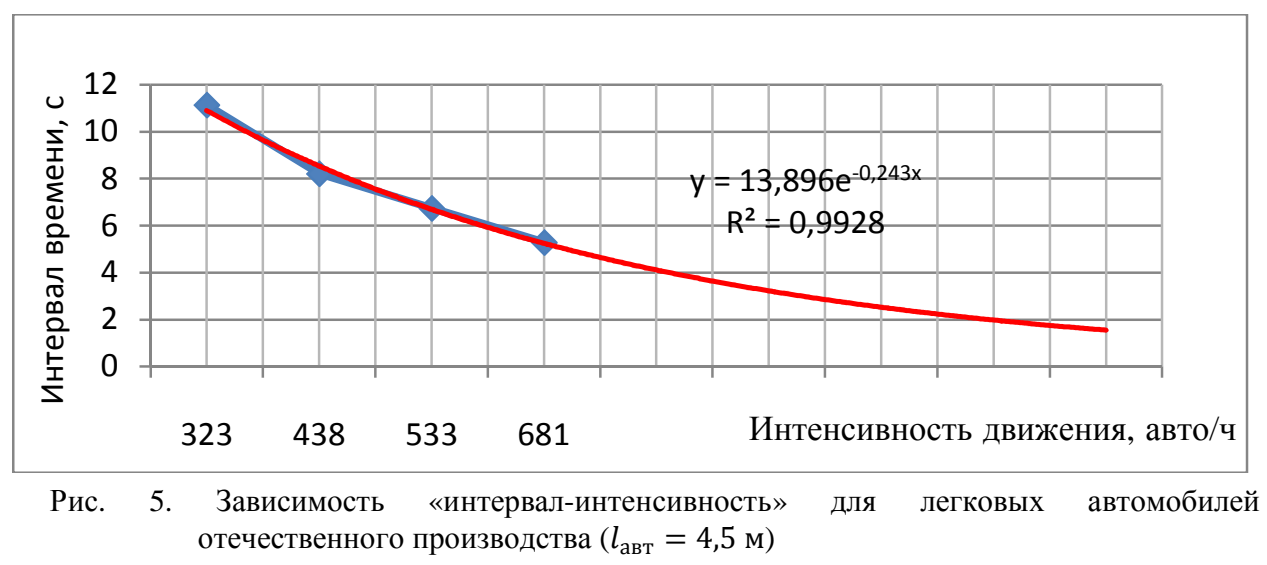




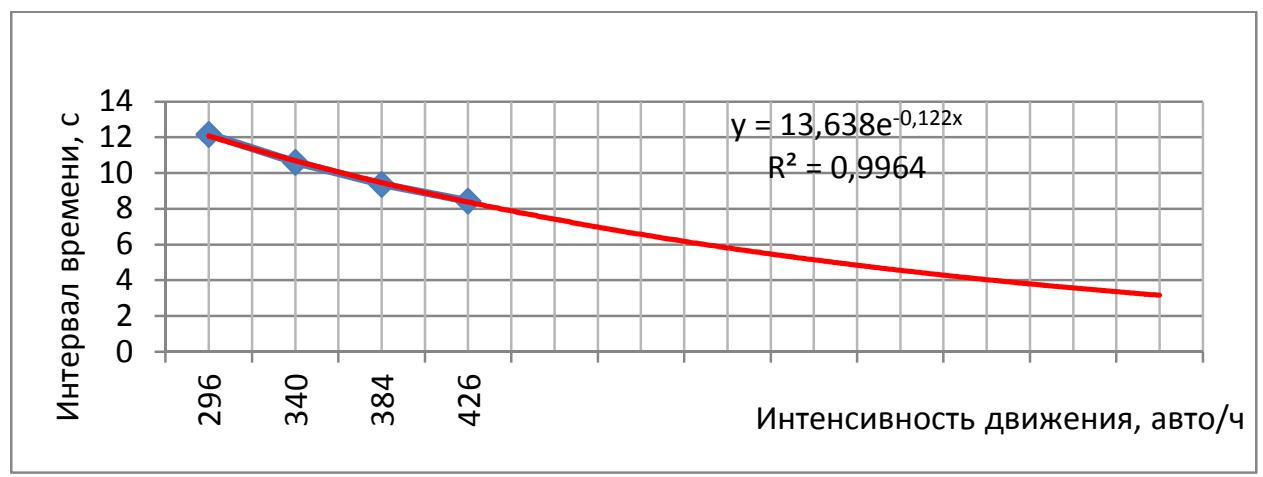

Рис. 6. Зависимость «интервал-интенсивность» для грузовых автомобилей $\left(l_{\text {авт }}=7,0\right.$ м)

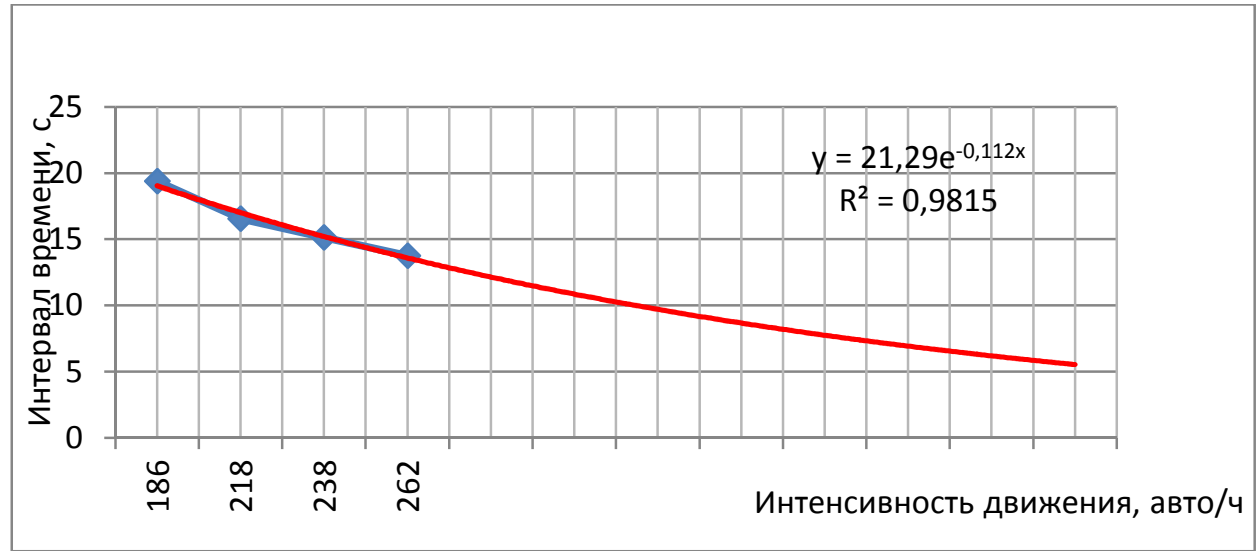

Рис.7. Зависимость «интервал-интенсивность» для автопоездов $\left(l_{\text {авт }}=12\right.$ м $)$

В результате исследования получено значение времени, необходимого для осуществления манёвра по смене полосы движения, в зависимости от интенсивности на съезде:

$$
T=19,142 \times e^{-0,078 \times N},
$$

где $\boldsymbol{T}$-необходимое время для осуществления манёвра, с;

$\boldsymbol{N}$ - интенсивность на съезде, авто / ч.

\section{4. ИССЛЕДОВАНИЕ ДЛИНЫ УЧАСТКА ДОРОГИ ДЛЯ СМЕНЫ ПОЛОСЫ ДВИЖЕНИЯ ПРИ НАЛИЧИИ ПЕРЕХОДНО-СКОРОСТНОЙ ПОЛОСЫ}

В зависимости от количества автомобилей в группе, определённого на основе системы массового обслуживания, и средней скорости транспортного потока на основной дороге, необходимая длина для удлинения переходно-скоростной полосы составит:

$$
S=v_{\text {гол }} \times \bar{n},
$$

где $v_{\text {гол }}-$ скорость движения транспортного потока на крайней полосе главной дороги, м/с; 
$\bar{n}-$ количество автомобилей в группе.

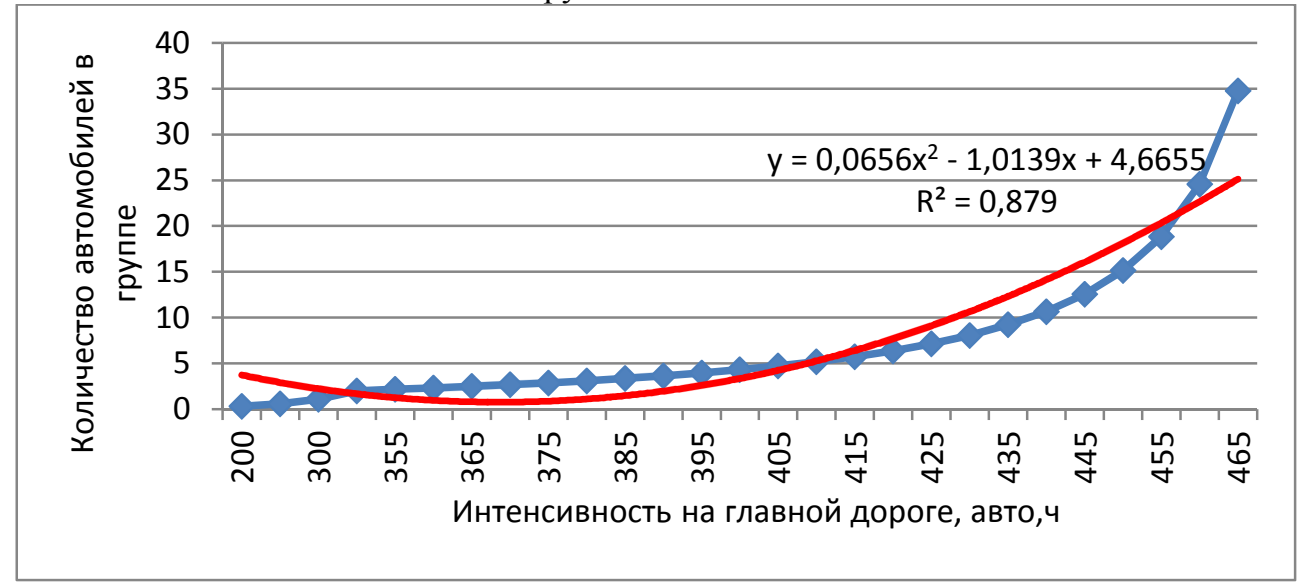

Рис. 8. График зависимости количества автомобилей в группе от интенсивности на основной дороге.

Согласно графика (рис. 8), построенного на основе системы массового обслуживания, согласно интенсивности движения на крайней полосе главной дороги, определяем количество автомобилей в группе. Согласно формуле (11), определяем необходимую длину переходно-скоростной полосы. Например, если интенсивность движения на крайней полосе главной дороги 400 авто / ч. при скорости движения автомобилей 60 км / ч (16,7 м / с), то количество автомобилей в группе - 5, в соответствии удлинить переходно-скоростную полосу необходимо на $5 \times 16,7=83 \mathrm{M}$.

\section{5. ВЫВОДЫ}

1. Разработана модель въезда автомобилей на основную (второстепенную) дорогу с переходно-скоростной полосы.

2. Исследовано время ожидания автомобилей для выполнения манёвра вливания в транспортный поток.

3. Разработаны уравнения для определения необходимой длины удлинения переходно-скоростной полосы для вливания автомобилей в основной поток.

\section{ЛИТЕРАТУРА}

[1] Довгополюк Л.А., Определение длины переходно-скоростных полос на дорожных развязках в разных уровнях, International Scientific and Practical Internet Conference. World Science September 22-24, 2014, UAE, Dubai 2014, C. 58-62.

[2] Довгополюк Л.А., Проектирование переходно-скоростных полос на дорожных развязках в разных уровнях, „Вестник. Научно-технический сборник” 2013/28, С. 160163.

[3] Довгополюк Л.А., Изменение полосы движения на автомобильной дороге, Материалы 70 науч. конф. профессорско-преподавательского состава, аспирантов, студентов и структурных подразделений университета: тезисы докладов, НТУ, Киев 2014, С. 170.

[4] Довгополюк Л.А., Необходимость определения времени ожидания при проезде дорожной развязки на разных уровнях, Материалы 68 науч. конф. профессорско- 
преподавательского состава, аспирантов, студентов и структурных подразделений университета: тезисы докладов, НТУ, Киев 2012, С. 158.

[5] Пальчик А.М., Транспортные потоки, НТУ, Киев 2010.

\section{DETERMINE THE LENGTH OF ACCELERATION AND DECELERATION LANES ON ROAD INTERCHANGES IN DIFFERENT LEVELS}

This paper presents a method for calculating the length of the acceleration and deceleration lanes at road junctions at different levels during acceleration. Increasing traffic on the roads calls for reconstruction of roads, part of which is the construction of road junctions at different levels. One of the main elements of the solution is the length of the acceleration and deceleration lanes during braking and acceleration of vehicles. Acceleration and deceleration lanes during braking serves as a release of the lane on the main road and reduce the speed of traffic on acceleration and deceleration lanes.

Speed change lane during acceleration is used to achieve an average speed equal to the speed on the main road and provides an infusion of cars with acceleration and deceleration lanes (CAP) in the lane on the main road. The main indicator is changing lanes on a PSP lane main road, which depends on the traffic on the main road and the time interval between cars. Performing maneuver the vehicle when changing lanes consists of the time for its implementation and long sections of road on which it is running. The paper presents the results of the study as time to perform the maneuver, and the length of the section of the road on which it is running. The results obtained are used for the design of road junctions at different levels, and to justify its type. With significant traffic on the main road there is a need lengthening the CAP, and if you can not enter - the construction of auxiliary lanes.

Keywords: road junctions at different levels, acceleration and deceleration lanes, lane, traffic composition, the average speed of traffic flow.

\section{DOI: 10.7862/rz.2015.mmr.6}

Tekst złożono w redakcji: styczeń 2015

Przyjęto do druku: marzec 2015 